\title{
Expression of Trefoil factor family peptides in the nasal allergic mucosa*
}

\author{
Nobuyuki Miyahara, Takashi Ishino, Takashi Kono, Keishin Go, Sachio Takeno, Rhinology 50: 408-416, 2012 \\ Masaya Takumida, Katsuhiro Hirakawa \\ DOI: 10.4193/Rhino11.221 \\ Department of Otorhinolaryngology, Head and Neck Surgery, Graduate School of Biomedical Sciences, Hiroshima University, \\ *Received for publication: \\ Hiroshima, Japan \\ October 12, 2011 \\ Accepted: June 20, 2012
}

\section{Summary}

Objective: Trefoil factor family (TFF) peptides are the secretory products of mucous cells and are closely associated with mucins. TFF peptides appear to be important in mucosal healing processes. Although TFF1 and TFF3 are expressed in the human respiratory tract, their role in the nasal mucosa is not thoroughly understood. We investigated the association between TFF peptides and mucins and the role TFF peptides in the human nasal mucosa.

Material and Methods: Patients undergoing turbinectomy were included and it was determined whether patients had nasal allergies or not. The localization of TFF1/3, MUC5AC/5B expression was investigated using immunohistochemistry. The levels of the mRNA transcripts were examined using quantitative real-time PCR.

Results:TFF1/3 had a similar pattern of localization in epithelial goblet cells and submucosal glandular cells. TFF1/3 co-localized with MUC5AC in the epithelium, and co-localized with MUC5B in the epithelium and the submucosal glandular cells. The levels of TFF1/3 and MUC5B mRNA in allergic patients were significantly increased.

Conclusion: Our results suggest that TFF1/3 may associate with MUC5AC and MUC5B in the nasal mucosa, and that up-regulation of TFF1/3 and MUC5B may play an important role in the clinical condition of the nasal allergic mucosa.

Key words: TFF1, TFF3, nasal allergy, nasal mucosa, mucin

\section{Introduction}

Mucins play an important role in maintaining the mucous membrane epithelium in the airways and gastrointestinal tract. Mucins in nasal mucosa are derived from two different cellular sources: the surface mucosal epithelium and submucosal glands (1). Mucus acts as a physical barrier to many harmful materials by preventing infectious agents from attaching to the respiratory epithelium and by inactivating various toxic materials ${ }^{(2)}$. The amount of mucus produced in the human airway is primarily balanced by the rate of production and clearance through resorption, vaporization, and ciliary transport ${ }^{(2)}$. Expression of mucins is greater in chronic airway diseases such as chronic sinusitis, allergic rhinitis, asthma, and cystic fibrosis than in normal airway mucosa ${ }^{(3-6)}$. In addition to mucins, peptides belonging to the trefoil factor family (TFF) have recently been reported as being present in the airway epithelium.

TFF peptides are secretory products of many mucous cells ${ }^{(1,7)}$. These peptides appear to be important in mucosal healing processes and neoplasia ${ }^{(8)}$. In humans, three TFF peptides have been described: TFF1 (pS2), TFF2 (formerly spasmolytic polypeptide SP), and TFF3 (intestinal trefoil factor, ITF or hP1.B). TFF peptides have been detected in the gastrointestinal tract ${ }^{\left({ }^{9}\right)}$, hypothalamus and pituitary glands ${ }^{(10)}$, conjunctiva ${ }^{(11)}$, uterus ${ }^{(12)}$, breast ${ }^{(13)}$, and also along mucosal surfaces of the human respiratory tract ${ }^{(7,14)}$.

The TFF peptides have a close association with mucins (9). In the gastrointestinal tract, TFF1 is associated with MUC6 expression, 


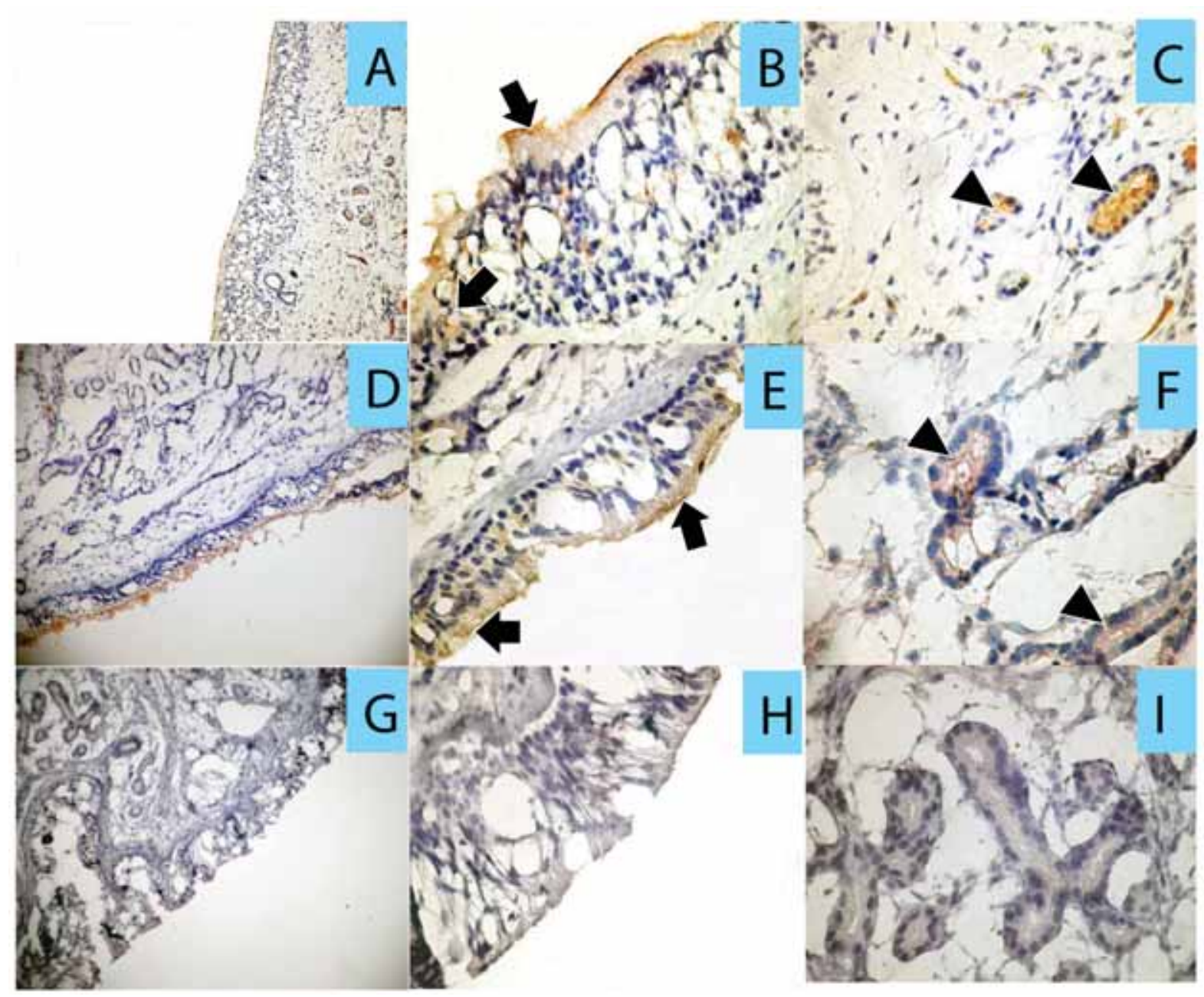

Figure 1. Immunostaining for TFF1and TFF3 in the nasal mucosa. (A) TFF1: The staining was positive in epithelial goblet cells and ciliated cells, and submucosal glandular cells (original magnification 100x). (B) TFF1: in epithelial goblet cells and ciliated cells (arrows) (original magnification 400x). (C) TFF1: in submucosal glandular cells (arrowhead) (original magnification 400X). (D) TFF3: The staining was positive in epithelial goblet cells and ciliated cells, and submucosal glandular cells (original magnification 100X). (E) TFF3: in epithelial goblet cells and ciliated cells (arrows) (original magnification 400X). (F) TFF3: in submucosal glandular cells (arrowhead) (original magnification 400x). Control specimens were incubated with Isotype Control For Rabbit Primary Ab(Invitrogen, 08-6199) or Isotype Control For Mouse Primary Ab(Invitrogen, 08-6599). Staining of control specimens was negative. (G) Control: (original magnification 100x) (H) Control: in the epithelium and (I) Control: in the submucosal glands (original magnification 400x).

TFF2 with expression of MUC5AC, and TFF3 with MUC2 expression ${ }^{(9)}$. The combination of TFF1+3 and MUC5AC was found in conjunctival goblet cells ${ }^{(11)}$. The combined action of TFF peptides and mucins are thought to play an important role in mucosal epithelial restitution and wound healing. Up-regulation of TFF peptides have an effect on ulceration and a variety of solid tumors ${ }^{(8,15)}$. TFF1 and TFF3 peptides are expressed, while

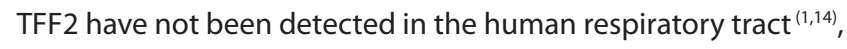
but their role in the nasal mucosa is not thoroughly understood. In this study, we examined the role of TFF peptides and their association with mucins in the human nasal mucosa.

\section{Materials and methods}

\section{Patients}

The study was carried out between October 2008 and January 2011 at the ENT Department of Hiroshima University Hospital, Hiroshima, Japan. Biopsies of the inferior turbinate mucosa were obtained from 28 patients ( 19 males and 9 females; age range 21 to 79 years) undergoing turbinectomy for the treatment of nasal obstruction. All patients had a nasal obstruction due to irreversible changes in the inferior turbinate or a deflected nasal septum. Patients with chronic sinusitis were excluded. No patients had received allergen-specific immunotherapy within the year before the study, and anti-allergic agents or corticosteroids 


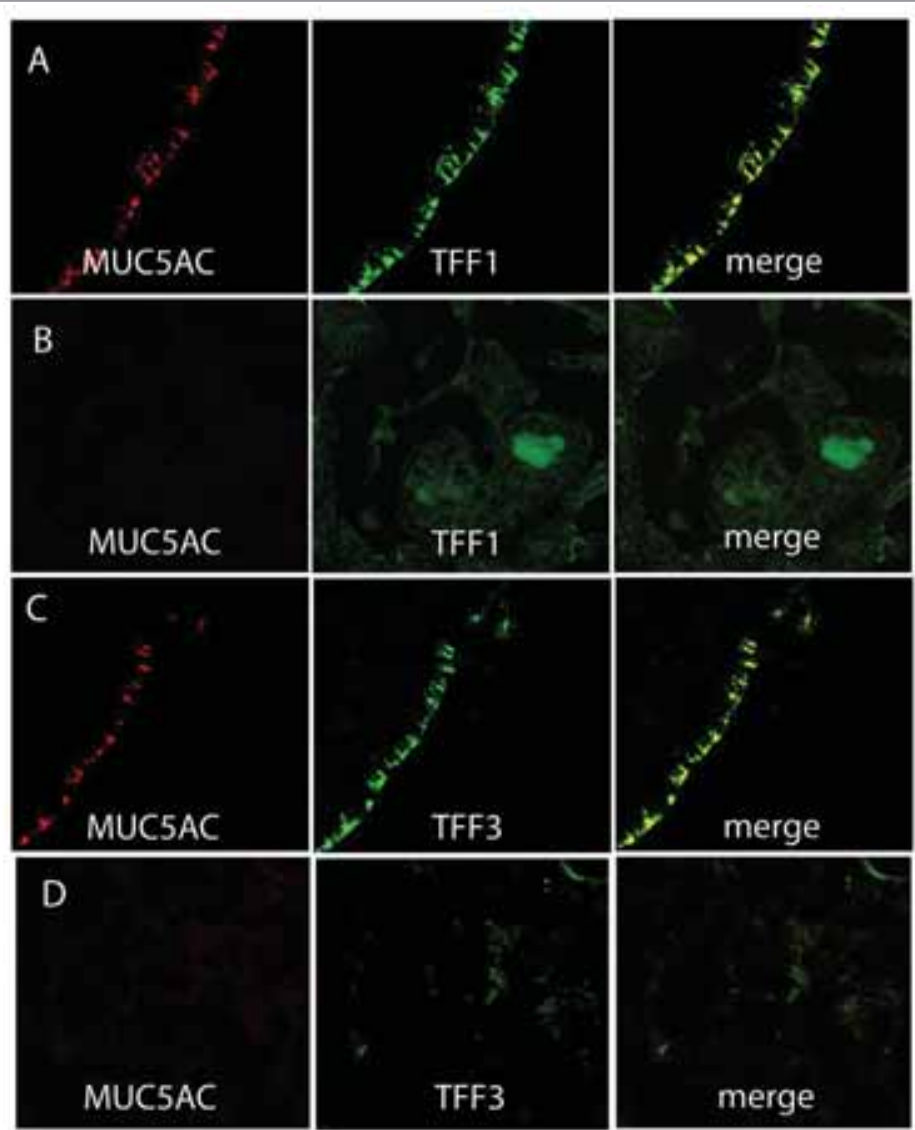

Figure 2. Double-staining for MUC5AC (red), TFF1 (green), and TFF3 (green) in the nasal mucosa. TFF1 co-localized with MUC5AC in the epithelium (A), but did not co-localize with MUC5AC in the submucosal glands (B). TFF3 co-localized with MUC5AC in the epithelium (C), but did not co-localize with MUC5AC in the submucosal glands (D) (original magnification 200x).

or antibiotics within the previous 2 months. We determined the nasal allergy status of all patients at the outset. Diagnosis of nasal allergy was made on the basis on nasal allergic symptoms and nasal allergic findings with specific IgE radioallergosorbent test (RAST). The criteria were based on a positive skin test for house dust mites, and a positive RAST score of 2 or more for Dermatophagoides farinae. Sixteen patients were diagnosed with nasal allergy ( 13 males and 3 females; age range 23 to 74 years) and 12 patients were nonallergic ( 6 males and 6 females; age range 21 to 79 years). The Institutional Review Board of the University of Hiroshima School of Medicine approved the study.

\section{Immunohistochemical staining}

Tissue specimens were fixed in Carnoy's fluid, dehydrated, frozen in OCT mounting medium (Sakura Finetechnical Co., Ltd., Tokyo, Japan), serially sectioned in $5 \mu \mathrm{m}$ increments, and mounted on glass slides. For antigen retrieval, sections were immersed in HistVT One (Nacalai Tesque, Kyoto, Japan) at $70^{\circ} \mathrm{C}$ for $40 \mathrm{~min}$. For peroxidase staining, sections were immersed in $0.3 \% \mathrm{H} 2 \mathrm{O} 2$ for $30 \mathrm{~min}$ at room temperature to block endogenous peroxidase activity. After pretreatment with blocking serum, specimens were incubated overnight with anti-TFF1 protein rabbit polyclonal antibody (1:100 dilution, Novocastra Laboratories Ltd., Newcastle upon Tyne, United Kingdom) or rabbit anti-TFF3 (1:50 dilution, Protein Tech Group, Inc., Chicago, IL, USA). Immunostaining was performed using the streptavidin-biotin amplification technique (ChemMate kit; DAKO, Glostrup, Denmark). Peroxidase activity was visualized by immersing tissue sections in diaminobenzidine (DAKO), resulting in a brown reaction product. Sections were then counterstained with Mayer's haematoxylin for $10 \mathrm{~min}$.

\section{Immunofluorescent double-staining}

For immunofluorescent staining of TFF1, TFF3, MUC5AC, and MUC5B, sections were labeled overnight at $4^{\circ} \mathrm{C}$ with primary antibodies to MUC5AC (mouse, 1:50 dilution, Novocastra Laboratories Ltd.) or MUC5B (mouse, 1:50 dilution, Chemicon International, Inc., Temecula, CA, USA). Then they were labeled with Alexa555 (anti-mouse) secondary antibody for $1 \mathrm{~h}$ at room temperature. Sections were then labeled with primary antibodies to TFF1 (rabbit, 1:100 dilution, Novocastra Laboratories Ltd.) and TFF3 (rabbit, 1:50 dilution, Protein Tech Group, Inc.) overnight at 


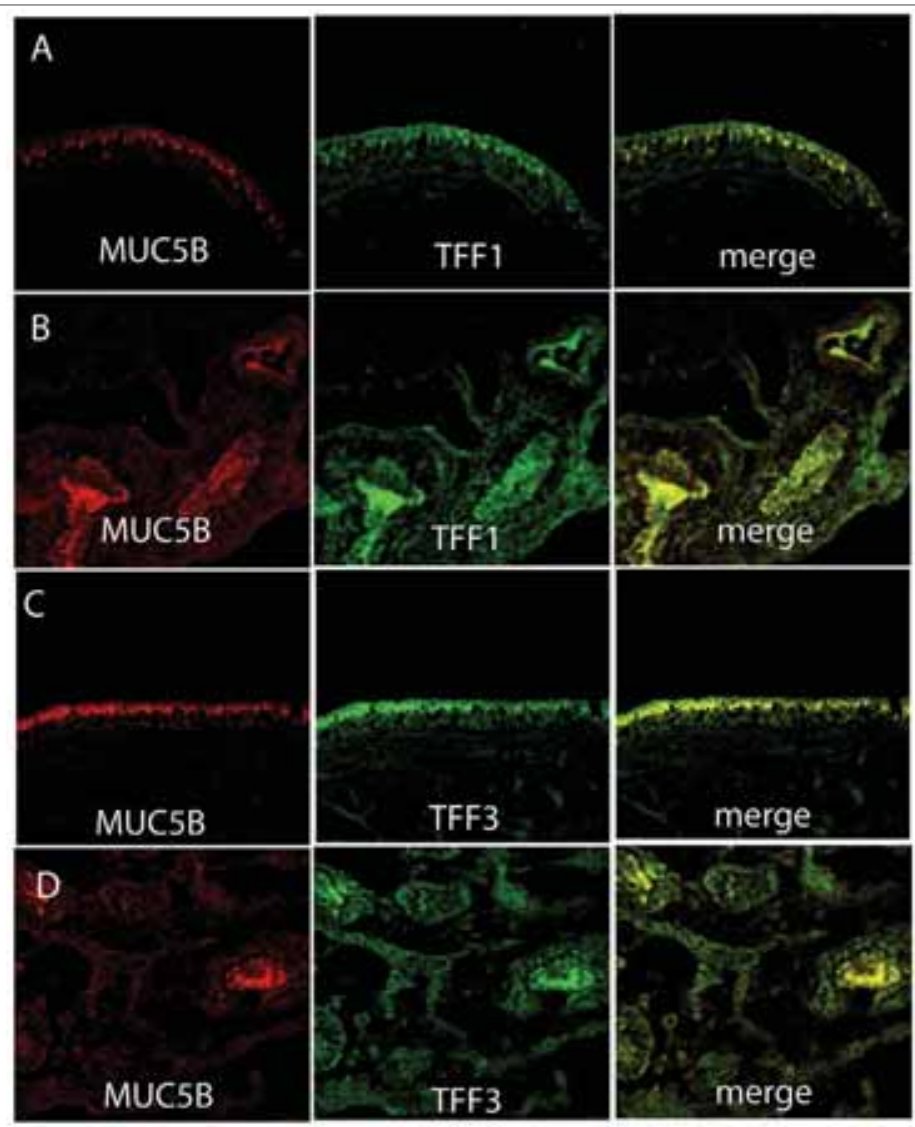

Figure 3. Double-staining for MUC5B (red), TFF1 (green), and TFF3 (green) in the nasal mucosa. TFF1 co-localized with MUC5B in the epithelium (A) and MUC5B in the submucosal glands (B). TFF3 co-localized with MUC5B in the epithelium (C) and MUC5B in the submucosal glands (D) (original magnification 200x).

$4^{\circ} \mathrm{C}$, followed by labeling with Alexa488 (anti-rabbit) secondary antibody for $1 \mathrm{~h}$ at room temperature. Finally, specimens were viewed with a confocal laser scanning microscope equipped with an appropriate filter set.

\section{Real-time PCR}

Tissue specimens were minced with scissors as soon as possible after retrieval, immediately treated in RNAlater (Ambion, Austin, TX, USA), and stored at $-20^{\circ} \mathrm{C}$. RNA was isolated using RNeasy mini kits (QIAGEN) according to the manufacturer's instructions. For CDNA synthesis, total RNA was transcribed with CDNA transcription reagents using a High Capacity RNA-to-cDNA kit (Applied Biosystems) according to the manufacturer's instructions. Gene expression was measured in real-time on a 7300 real-time PCR system (Applied Biosystems) using TaqMan Gene Expression Assays. The absolute values for TFF1 (Hs00907239_ml), TFF3 (Hs00173625_ml), MUC5AC (Hs01365616_ml), and MUC5B (Hs00861595_ml) were normalized to the value of GAPDH (Hs99999905_ml), and the relative value from the vehicle-treated control group was designated as one arbitrary unit.

\section{Statistical analysis}

We assumed that the data were not normally distributed; therefore, comparisons of gene expression levels between clinical groups were made using the non-parametric Mann-Whitney U-test. A p-value less than 0.05 was considered to be statistically significant. The non-parametric Spearman rank correlation test was used to detect correlations of paired data.

\section{Results}

Immunohistochemistry

All tissue sections (from patients both with and without nasal allergy) were TFF1 and TFF3 immunoreactive. The localization pattern of both TFF1 and TFF3 were similar in epithelial goblet cells, ciliated cells, and submucosal glandular cells (Figure 1). Control specimens were incubated with Isotype Control For Rabbit Primary Ab (Invitrogen, 08-6199) or Isotype Control For Mouse Primary Ab (Invitrogen, 08-6599), and the staining of these specimens was negative.

In the double-staining study, TFF1 and TFF3 co-localized with MUC5AC in the epithelium, but staining was absent or weak in 


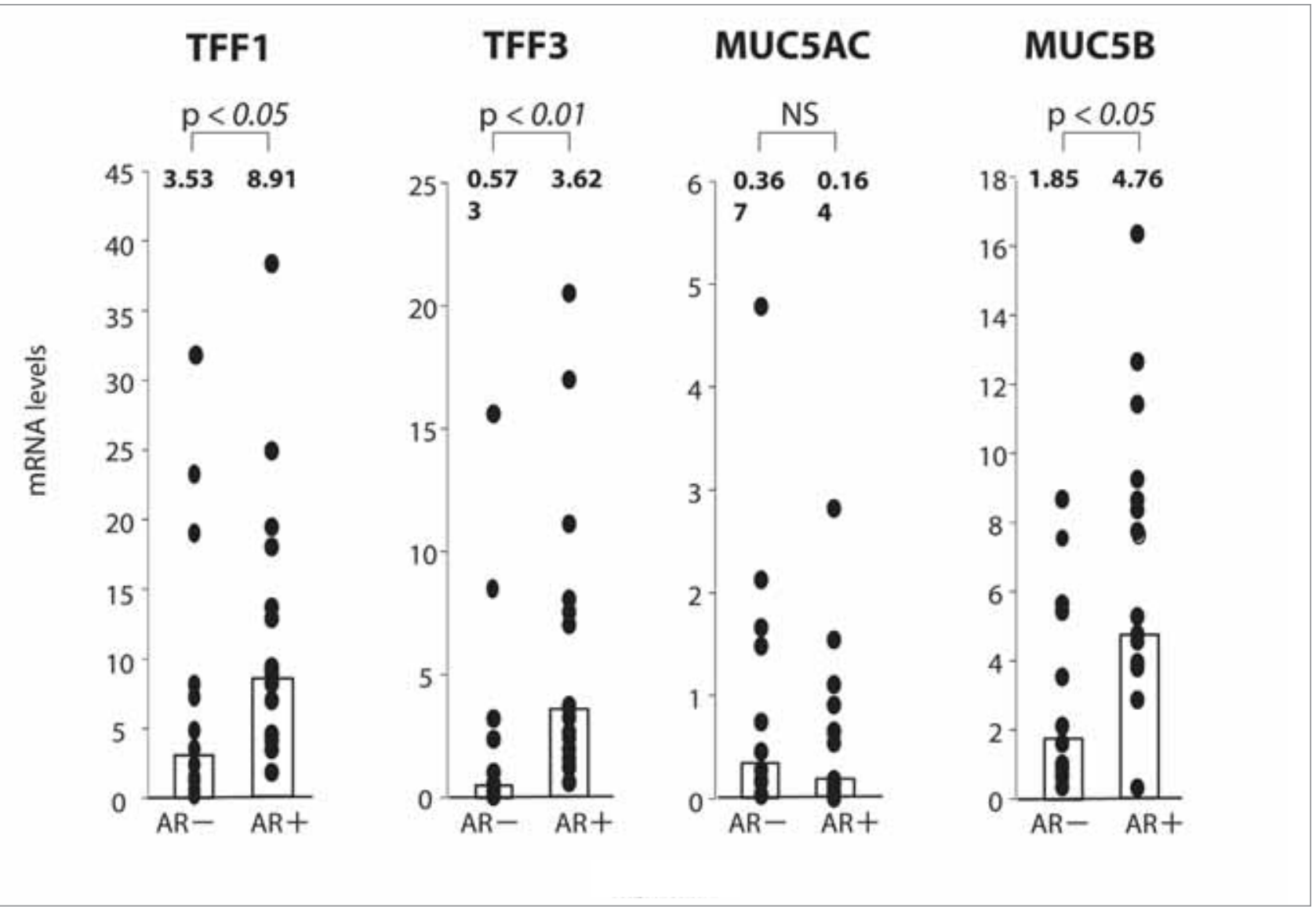

Figure 4. We normalized the values for expression of all target genes to expression of GAPDH in all tissues, and compared these values to the expression levels of TFF1, TFF3, MUC5AC, and MUC5B in the nasal mucosa of allergic (AR+) and nonallergic (AR-) patients. NS means'not significant'. The expression of TFF1 mRNA was significantly higher in the nasal mucosa of allergy patients compared to nonallergic patients $(p<0.05)$. The expression of TFF3 mRNA was significantly higher in the nasal mucosa of allergy patients compared to nonallergic patients $(p<0.01)$. The expression of MUC5AC mRNA was not significantly different between allergic and nonallergic patients. The expression of MUC5B mRNA was significantly higher in the nasal mucosa of allergic patients compared to nonallergic patients $(p<0.05)$. Columns represent median values.

the submucosal glands. TFF1 and TFF3 co-localized with MUC5B in the epithelium and the submucosal glands (Figures 2 and 3). Control specimens were incubated with Isotype Control For Rabbit Primary Ab (Invitrogen, 08-6199) or Isotype Control For Mouse Primary Ab (Invitrogen, 08-6599), and staining of these specimens was negative (data not shown).

Real-time PCR

Quantitative real-time PCR was conducted to determine TFF1, TFF3, MUC5AC, and MUC5B mRNA transcript levels. We normalized the values of all target genes in all tissues to GAPDH and compared target gene expression to the expression of TFF1, TFF3, MUC5AC, and MUC5B in the inferior turbinate mucosa (Figure 4).

TFF1 The expression of TFF1 mRNA was significantly higher in the inferior turbinate of specimens from nasal allergy patients compared to those from non-allergy patients $(p<0.05)$.
TFF3 The expression of TFF3 mRNA was significantly higher in the inferior turbinate of specimens from nasal allergy patients compared to those from non-allergy patients $(p<0.01)$.

MUC5AC The expression of MUC5AC mRNA in the inferior turbinate of both nasal allergic and nonallergic specimens was not significantly different.

MUC5B The expression of MUC5B mRNA in the inferior turbinate of patients with nasal allergy was significantly higher compared to non-allergy patients $(p<0.05)$. We also examined whether TFF1/3 and MUC5B mRNA expression values correlated between patients and found that there was a statistically significant correlation between TFF1/3 and MUC5B expression (Figures 5 and 6).

\section{Discussion}

TFF peptides are secretory products of many mucous cells ${ }^{(1,7)}$. In humans, three trefoil factor peptides (TFF1, TFF2, and TFF3) that 


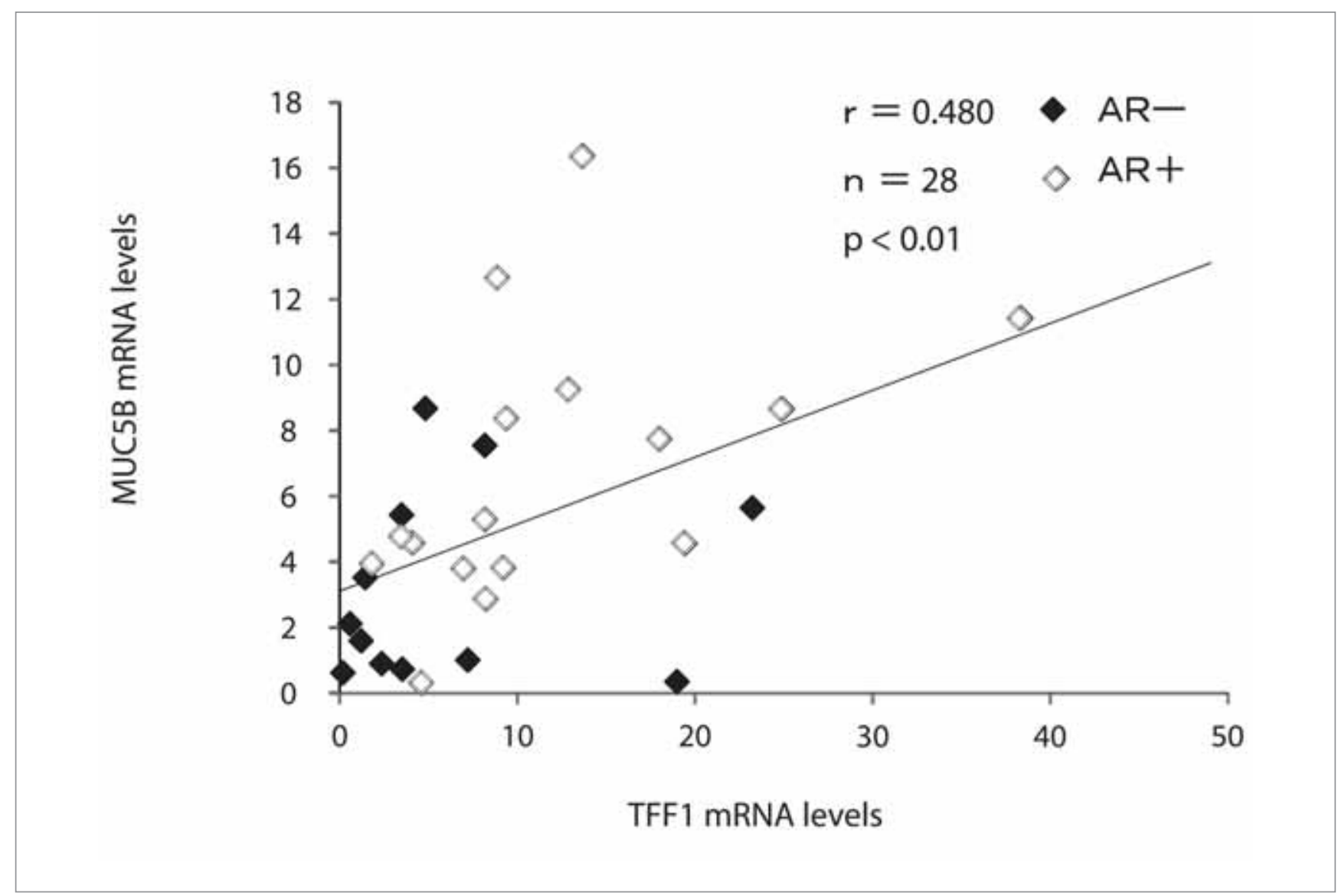

Figure 5. Correlation of mRNA expression values for TFF1 and MUC5B in the nasal mucosa of allergic (AR+) and nonallergic (AR-) patients.

have an organ-specific expression have been described. The primary TFF1 expression sites are gastric foveolar cells, while TFF2 is found in the deeper glands of the stomach and duodenum and TFF3 is largely restricted to intestinal goblet cells ${ }^{(16)}$. The TFF peptides appear to be important in mucosal healing processes and neoplasia ${ }^{(8)}$. Deletion of TFF genes in mice leads to atrophy of the gastric mucosa and impaired wound healing in the intestinal mucosa ${ }^{(17,18)}$. Conversely, administration of TFF has been shown to lead to wound healing in the intestinal mucosa ${ }^{(15,19)}$.

Some researchers have reported detecting TFF1 and TFF3 in airways. TFF1 expression has been reported in small cell lung carcinomas, bronchial carcinoids, and adeno-carcinomas ${ }^{(20,21)}$. High TFF3 mRNA levels were observed in the HC-12 lung cell line (16). In addition, TFF1 and TFF3 are expressed and secreted in normal and inflamed airways. Several reports have demonstrated the presence of TFF 1 and TFF3 in the nasal mucosa and polyps ${ }^{(1,7,14)}$. In the inferior turbinate of patients with no history of nasal infection or allergy, TFF1 and TFF3 mRNA are localized in epithelial cells and the submucosal glandular epithelium (1). Silva et al., reported that TFF1 and TFF3 localize in the goblet and ciliated cells, as well as in some submucosal cells of normal and inflamed airways ${ }^{(14)}$, and that TFF peptides might influence important bacterial/host interactions, such as adhesion of bacteria to host cells, phagocytosis, and immune signaling ${ }^{(14)}$. Some reports have also shown that TFF peptides are believed to regulate mucosal differentiation processes. In a murine asthma model, Kouznetsova et al. indicated that TFF1 might play an intrinsic role as an autocrine factor for the trans-differentiation of Clara cells into goblet cells ${ }^{(22)}$. LeSimple et al. proposed that TFF3 might be able to induce ciliogenesis and promote epithelial ciliated cell differentiation in nasal polyps and bronchi when the airway epithelium is damaged ${ }^{(7)}$.

In our immunohistochemistry study, TFF1 and TFF3 localized similarly in specimens from the nasal mucosa of both allergic and nonallergic patients. The localization patterns of TFF1 and TFF3 were similar in epithelial goblet cells, ciliated cells, and submucosal glandular cells. Real-time PCR revealed that both TFF1 and TFF3 were up-regulated in allergic patients. These results suggest that up-regulation of TFF1/3 may be associated with epithelial cell differentiation in the nasal mucosa of patients with allergic rhinitis. Cui reported that allergen exposure leaded to a possible transdifferentiation of $\mathrm{CC} 10$ secreting cells into TFF1 secreting cells and/or goblet cells in upper airways ${ }^{(23)}$. Iwakiri et al. reported that TFF3 plays a central role in promoting differentiation to goblet cells in the intestine ${ }^{(24)}$. In the respiratory tract, LeSimple et al. showed that there was a tendency toward a 


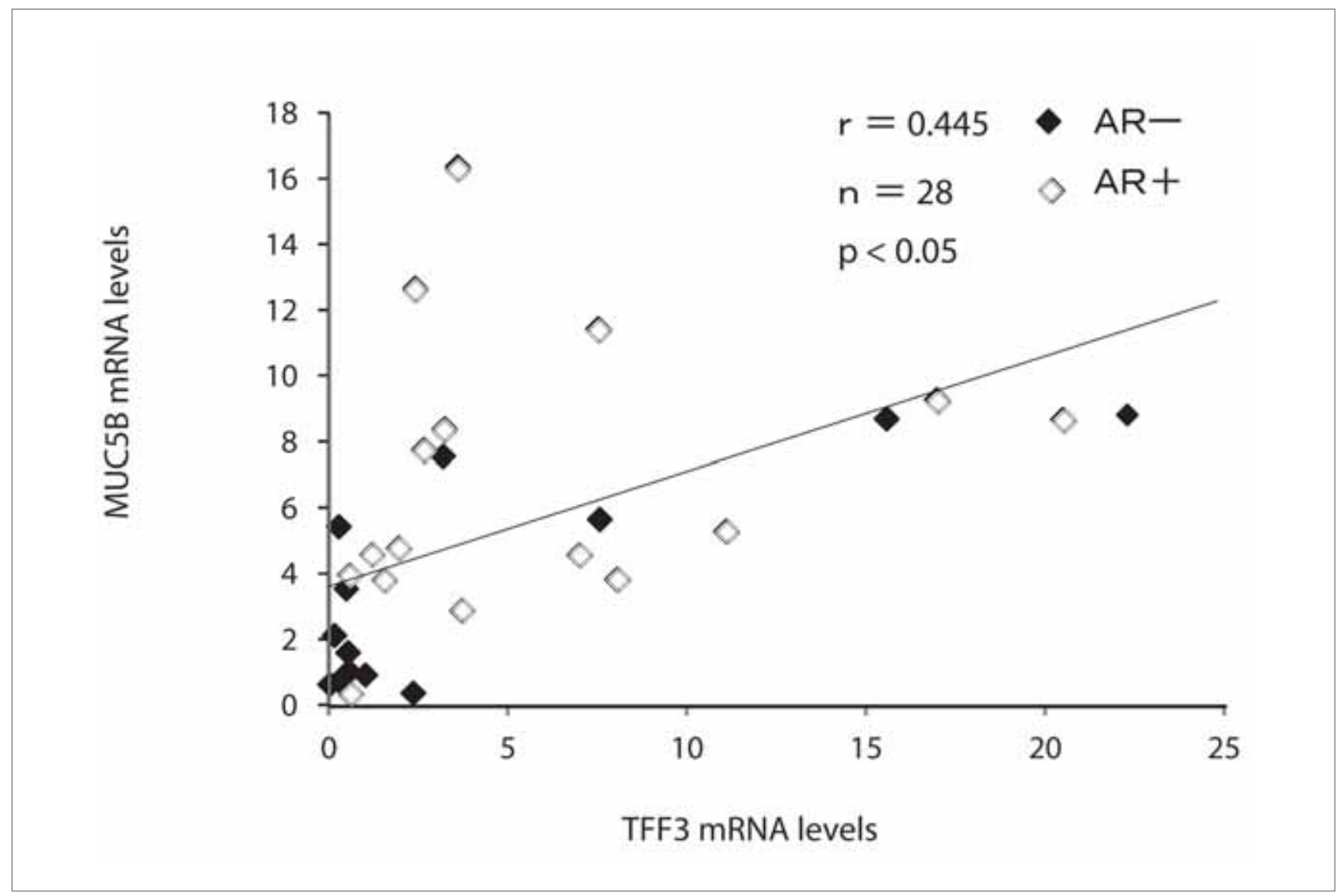

Figure 6. Correlation of mRNA expression values for TFF3 and MUC5B in the nasal mucosa of allergic (AR+) and nonallergic (AR-) patients.

high number of goblet cells upon TFF3 stimulation ${ }^{(7)}$. Therefore, in allergic conditions, TFF1/3 may induce goblet cell hyperplasia and lead to mucus overproduction. We propose that TFF1/3 may regulate mucosal differentiation processes in the nasal mucosa, similarly in nasal polyps and bronchi.

TFF peptides are closely associated with mucins ${ }^{(9)}$. TFF peptides in combination with mucins contribute to mucosal defense. In the gastrointestinal tract, TFF1 is associated with MUC6 expression, TFF2 with MUC5AC expression, and TFF3 with expression of MUC2 ${ }^{(9)}$. The combination of TFF1+3 and MUC5AC was found in conjunctival goblet cells ${ }^{(11)}$. Both MUC5AC and MUC5B are important in maintaining the mucous membrane epithelium of airways, and expression of these proteins increases in chronic inflammatory airway diseases such as chronic sinusitis and asthma ${ }^{(3-5,25-27)}$. Mucins, including MUC5AC and MUC5B, induce nasal obstruction and dyspnea by interrupting the airflow in the airway and also aggravate coughing by promoting the adhesion of inspired noxious substances to the airway mucosa (2). Six mucin genes (MUC1, MUC2, MUC4, MUC5AC, MUC5B, and MUC7) are expressed in the human nasal mucosa ${ }^{(28)}$. Mucin MUC5AC is present in sinus epithelial goblet cells but is absent or weakly expressed in submucosal glands, while immunoreactivi- ty indicative of MUC5B expression is localized in the goblet cells and mucous cells of submucosal glands ${ }^{(3,4)}$. Lee et al. reported that the localization of TFF1 and TFF3 corresponded with that of MUC1, MUC2, MUC4, and MUC5AC in nasal mucosa epithelial cells ${ }^{(1)}$. Wiede et al. reported that TFF3 colocalized primarily with MUC5B in submucosal gland mucous cells, and observed both TFF3 and MUC5AC together in respiratory goblet cells in the lower respiratory tract ${ }^{(29)}$. LeSimple et al. reported that MUC5AC is expressed in the secretory granules of all the goblet cells they observed, whereas the anti-TFF3 antibody stained a subcategory of MAC5AC-positive cells and there was partial overlap between cells that expressed TFF3 and MUC5B ${ }^{(7)}$.

Our immunofluorescence double-staining results showed that TFF1 and TFF3 co-localize with MUC5AC in the epithelium, and that TFF1 and TFF3 co-localize with MUC5B in both the epithelium and the submucosal glands. Expression of TFF1/3 and MUC5B was significantly higher in the inferior turbinate of patients with nasal allergy compared to patients without allergies. In addition, statistically significant correlation was found between expression TFF1/3 and MUC5B. A previous report commented that TFF3 is co-secreted with different mucins depending on the particular cell type ${ }^{(29)}$. Our results showed 
that in the upper respiratory tract, colocalization of TFF1/3 and MUC5AC/5B followed a pattern similar to that reported by Lee et al. in their study of the nasal mucosa and to the study by Wiede et al. of the lower respiratory tract. Our results indicate that upregulation of TFF1/3 and MUC5B may play an important role in the mucosa of patients suffering nasal allergy.

Respiratory mucus overproduction with an up-regulation of mucin gene expression is one of the major manifestations of chronic airway diseases ${ }^{(3,5,6)}$. Mucins are synthesized primarily by two different cell types in the airway tract: goblet cells and submucosal glandular cells. The amount of mucus produced in the human airway is normally balanced by the rates of production and clearance through resorption, vaporization, and ciliary transport (2). Overexpression of mucins and goblet cell hyperplasia are known to occur in asthma patients, leading to airway obstruction and dyspnea ${ }^{(30,31)}$. The same conditions affect the nasal mucosa in allergic rhinitis, and Nakaya et al. reported that allergen challenge causes goblet cell hyperplasia in the nasal airway $^{(32)}$.

Our study showed that there is significant overproduction of both TFF1/3 and MUC5B in allergic rhinitis, which is consistent with previous reports indicating that TFF peptides regulate mucosal differentiation processes in the respiratory tract ${ }^{(7,22)}$. These results suggest that goblet cell hyperplasia might be induced by TFF $1 / 3$ overexpression, thereby leading to overexpression of MUC5B in the allergic nasal mucosa. Therefore, regulating TFF might in turn regulate goblet cell hyperplasia and reduce the overexpression of mucins in the allergic nasal mucosa, improving symptoms such as nasal obstruction and dyspnea. Our results suggest that TFF peptides are promising targets for the treatment of chronic and acute airway diseases via pharmaceutical interventions ${ }^{(33)}$.

\section{Conclusions}

This study demonstrates that TFF 1 and TFF3 are expressed in the nasal mucosa and that their expression may be associated with expression of the mucins MUC5AC and MUC5B. The levels of TFF $1 / 3$ and MUC5B were significantly higher in the inferior turbinate of patients with nasal allergy compared to nonallergic patients. Up-regulation of TFF1/3 and MUC5B suggests that TFF peptides may play an important role in the clinical condition of the nasal mucosa in allergic patients. However, there was no sufficient evidence about TFF roles in nasal mucosa. Therefore, we require further investigation into the roles of TFF peptides in the nasal mucosa.

\section{Acknowledgements}

This study was carried out with the kind cooperation of the Analysis Center of Life Science, Hiroshima University. We thank Ms. Ai Kashima for excellent technical assistance.

\section{References}

1. Lee $\mathrm{SH}$, Lee $\mathrm{SH}$, Oh BH, Lee HM, Choi JO Jung KY. Expression of mRNA of trefoil factor peptides in human nasal mucosa. Acta Otolaryngol. 2001; 121: 849-853.

2. Yoon JH, Park IY. Mucin gene expression and mucin secretion in human airway epithelium. Rhinology. 1998; 36: 146-152.

3. Kim DH, Chu HS, Lee JY, Hwang SJ, Lee SH, Lee HM. Up-regulation of MUC5AC and MUC5B mucin genes in chronic rhinosinusitis. Arch Otolaryngol Head Neck Surg. 2004; 130: 747-752

4. Ding GQ, Zheng CQ. The expression of MUC5AC and MUC5B mucin genes in the mucosa of chronic rhinosinusitis and nasal polyposis. Am J Rhinol. 2007; 21: 359-366.

5. Groneberg DA, Eynott PR, Lim S, Oates T, Wu R, Carlstedt I. Expression of respiratory mucins in fatal status asthmaticus and mild asthma. Histopathology. 2002; 40: 367-373.

6. Voynow JA, Selby DM, Rose MC. Mucin gene expression (MUC1, MUC2, and MUC5/5AC) in nasal epithelial cells of cystic fibrosis, allergic rhinitis, and normal individuals. Lung. 1998; 176: 345-354.

7. LeSimple P, van Seuningen I, Buisine MP, Copin MC, Hinz M, Hoffmann W, et al. Trefoi factor family 3 peptide promotes human airway epithelial ciliated cell differentiation. Am J Respir Cell Mol Biol. 2007; 36: 296-303.

8. Longman RJ, Thomas MG, Poulsom R. Trefoil peptides and surgical disease. Br J Surg. 1999; 86: 740-748

9. Wong WM, Poulsom R, Wright NA. Trefoil peptides. Gut. 1999; 44: 890-895.

10. Probst JC, Zetzsche T, Weber M, Theilemann P, Skutella T, Landgraf R, et al. Human intestinal trefoil factor is expressed in human hypothalamus and pituitary: evidence for a novel neuropeptide. FASEB J. 1996; 10: 1518-1523.

11. Langer G, Jagla W, Behrens-Baumann W, Walter S, Hoffmann W. Secretory peptides TFF1 and TFF3 synthesized in human conjunctival goblet cells. Invest Ophthalmol Vis Sci. 1999; 40: 2220-2224.

12. Hauser F, Poulsom R, Chinery R, Rogers LA, Hanby AM, Wright NA, et al. hP1.B, a human P-domain peptide homologous with rat intestinal trefoil factor, is expressed also in the ulcer-associated cell lineage and the uterus. Proc Natl Acad Sci USA. 1993; 90: 6961-6965.

13. Poulsom R, Hanby AM, Lalani EN, Hauser F, Hoffmann W, Stamp GW. Intestinal tre- foil factor (TFF 3) and pS2 (TFF 1), but not spasmolytic polypeptide (TFF 2) mRNAs are co-expressed in normal, hyperplastic, and neoplastic human breast epithelium. J Pathol. 1997; 183: 30-38.

14. dos Santos Silva E, Ulrich M, Döring G, Botzenhart K, Gött P. Trefoil factor family domain peptides in the human respiratory tract. J Pathol. 2000; 190: 133-142.

15. Babyatsky MW, deBeaumont M, Thim L, Podolsky DK. Oral trefoil peptides protect against ethanol- and indomethacin-induced gastric injury in rats. Gastroenterology. 1996; 110: 489-497.

16. May FE, Westley BR. Expression of human intestinal trefoil factor in malignant cells and its regulation by oestrogen in breast cancer cells. J Pathol. 1997; 182: 404-413.

17. Mashimo H, Wu DC, Podolsky DK, Fishman MC. Impaired defense of intestinal mucosa in mice lacking intestinal trefoil factor. Science. 1996; 274: 262-265.

18. Playford RJ, Marchbank T, Goodlad RA, Chinery RA, Poulsom R, Hanby AM. Transgenic mice that overexpress the human trefoil peptide pS2 have an increased resistance to intestinal damage. Proc Natl Acad Sci USA. 1996; 93: 2137-2142. 
19. Cook GA, Thim L, Yeomans ND, Giraud AS Oral human spasmolytic polypeptide protects against aspirin-induced gastric injury in rats. J Gastroenterol Hepatol. 1998; 13: 363-370.

20. Wang DG, Johnston CF, Liu WH, Sloan JM, Buchanan KD. Expression of a breast-cancer-associated protein (pS2) in human neuro-endocrine tumours. Int J Cancer. 1997 74: 270-274.

21. Luqmani YA, Ryall G, Shousha S, Coombes RC. An immunohistochemical survey of pS2 expression in human epithelial cancers. Int J Cancer 1992: 50: 302-304

22. Kouznetsova I, Chwieralski CE, Bälder R, Hinz M, Braun A, Krug N, et al. Induced trefoil fac tor family 1 expression by trans-differentiating Clara cells in a murine asthma model. Am J Respir Cell Mol Biol. 2007; 36: 286-295.

23. Cui YH, Wang YY, Liu Z. Transdifferentiation of Clara cell 10-kDa protein secreting cells in experimental allergic rhinitis. Am J Rhinol Allergy. 2011: 25: 145-151.

24. Iwakiri D, Podolsky DK. Keratinocyte growth factor promotes goblet cell differentiation through regulation of goblet cell silencer inhibitor. Gastroenterology. 2001; 120: 13721380
25. Hovenberg HW, Davies JR, Herrmann A Lindén CJ, Carlstedt I. MUC5AC, but not MUC2, is a prominent mucin in respiratory secretions. Glycoconj J. 1996; 13: 839-847.

26. Thornton DJ, Carlstedt I, Howard M, Devine $\mathrm{PL}$, Price MR, Sheehan JK. Respiratory mucins: identification of core proteins and glycoforms. Biochem J. 1996; 316: 967-975.

27. Thornton DJ, Howard M, Khan N, Sheehan JK. Identication of two glycoforms of the MUC5B mucin in human respiratory mucus: evidence for a cysteine-rich sequence repeated within the molecule. J Biol Chem 1997: 272: 9561-9566.

28. Aust MR, Madsen CS, Jennings $A$ Kasperbauer JL, Gendler SJ. Mucin mRNA expression in normal and vasomotor inferior turbinates. Am J Rhinol. 1997; 11: 293 302

29. Wiede A, Jagla W, Welte T, Köhnlein T, Busk H, Hoffmann W. Localization of TFF3, a new mucus-associated peptide of the human respiratory tract. Am J Respir Crit Care Med 1999; 159: 1330-1335.

30. Izuhara K, Ohta S, Shiraishi H, Suzuki S, Taniguchi K, Toda S, et al. The mechanism of mucus production in bronchial asthma. Curr Med Chem. 2009; 16: 2867-2875.
31. Morcillo EJ, Cortijo J. Mucus and MUC in asthma. Curr Opin Pulm Med. 2006; 12: 1-6.

32. Nakaya M, Dohi M, Okunishi K, Nakagome K, Tanaka R, Imamura M, et al. Prolonged allergen challenge in murine nasal allergic rhinitis: nasal airway remodeling and adaptation of nasal airway responsiveness. Laryngoscope. 2007; 117: 881-885.

33. Hoffmann W. TFF (trefoil factor family) peptides and their potential roles for differentiation processes during airway remodeling. Curr Med Chem. 2007; 14: 2716-2719.

\section{Nobuyuki Miyahara}

1-2-3, Kasumi, Minami-ku

Hiroshima 734-8551

Japan

Tel: +81-082-2575252

Fax: +81-082-2575254

E-mail: d063494@hiroshima-u.ac.jp

\section{ADVERTISEMENT}

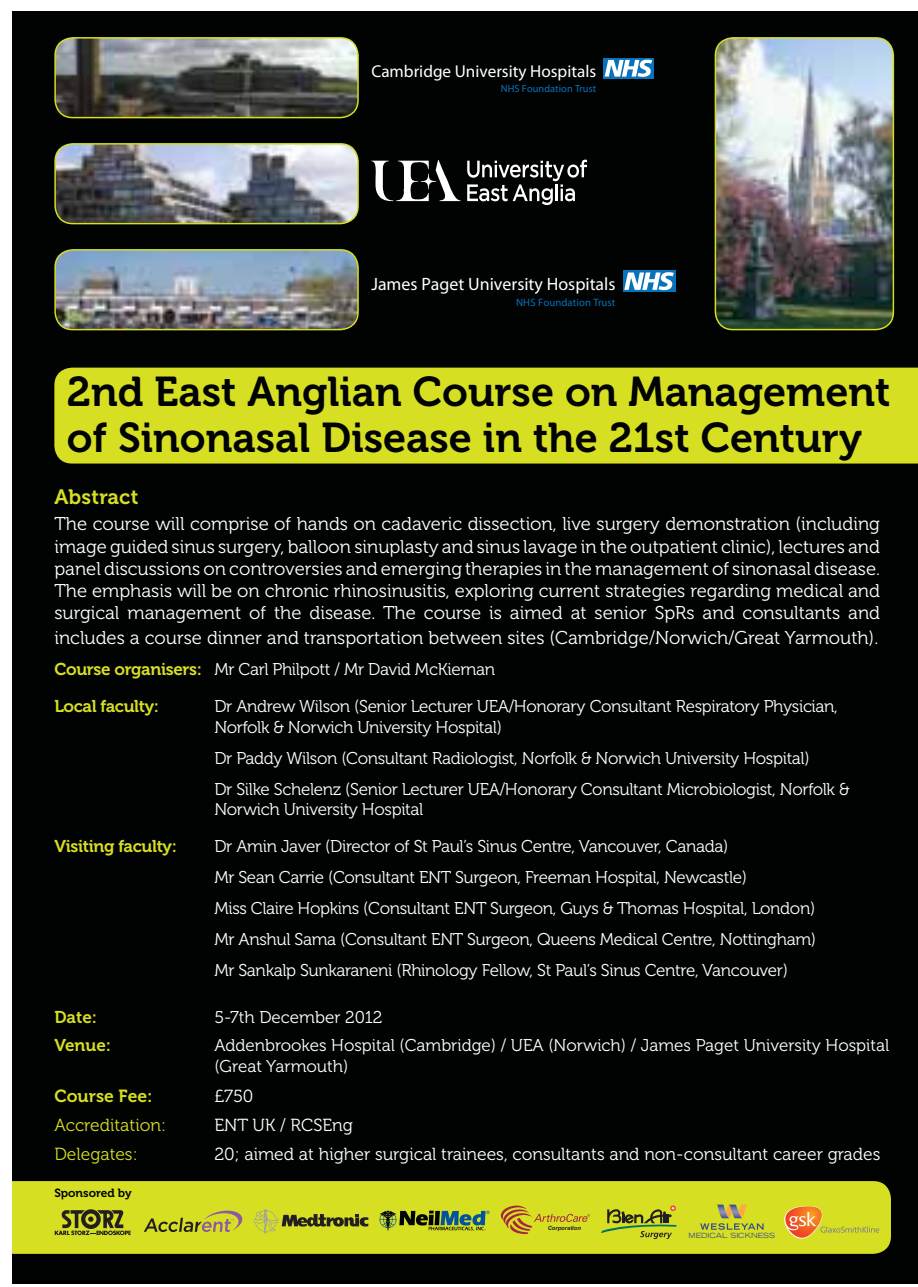

